\title{
IL-33 notably inhibits the growth of colon cancer cells
}

\author{
XUHUI CHEN $^{1,2^{*}}$, KUANCHANG LU $^{2 *}$, NOAH J. TIMKO ${ }^{3}$, DYLAN M. WEIR ${ }^{3}$, ZIWEN ZHU ${ }^{3}$, \\ CHENGLU QIN $^{3}$, JEFFERY D. MANN ${ }^{2}$, QIAN BAI ${ }^{3}$, HUAPING XIAO ${ }^{2,4}$, MICHAEL B. NICHOLL $^{3}$, \\ MARK R. WAKEFIELD ${ }^{3}$ and YUJIANG FANG ${ }^{2,3}$ \\ ${ }^{1}$ Department of Surgery, Luohu Hospital, Shenzhen, Guangdong 518000, P.R. China; \\ ${ }^{2}$ Department of Microbiology, Immunology and Pathology, Des Moines University College of Osteopathic Medicine, \\ Des Moines, IA 50312; ${ }^{3}$ Department of Surgery, University of Missouri School of Medicine, Columbia, MO 65212, USA; \\ ${ }^{4}$ The Affiliated Hospital of Xiangnan University, Chenzhou, Hunan 423000, P.R. China
}

Received November 10, 2017; Accepted March 15, 2018

DOI: $10.3892 / 01.2018 .8728$

\begin{abstract}
Interleukin-33 (IL-33), a damage-associated molecular pattern molecule, is a cytokine within the IL-1 interleukin family that binds to the plasma membrane receptor suppression of tumorigenicity 2 on numerous cell types. IL-33 has been extensively studied in its role in autoimmune diseases, host responses to pathogens and allergens, and has been associated with tumorigenic effects in cancer research. The present study was performed to investigate the effects of IL-33 on colon cancer cells, based off the previous data that have demonstrated an anti-tumor effect of IL-33 on pancreatic cancer cells. The effects of IL-33 on proliferation, cell survival and apoptosis on human HCT-116 colon cancer cells were examined using clonogenic survival assays, proliferation and caspase-3 activity kits, terminal deoxynucleotidyl transferase-mediated dUTP nick-end labeling staining and immunocytochemistry. It was determined that the HCT-116 cells demonstrated an notable decrease in optical density value upon incubation with IL-33, along with a decrease in the number of colonies, compared with the controls. It was further determined that the anti-proliferative effect of IL-33 on HCT-116 cells was associated with downregulation of the pro-proliferative molecules cyclin $\mathrm{B}$, cyclin $\mathrm{D}$ and cyclin dependent kinase 2. An apoptosis-inducing effect of IL-33 on HCT-116 cells was associated with downregulation of the anti-apoptotic molecules Flice-like inhibitory protein and B-cell lymphoma 2. Taken together, the results indicated that IL-33 inhibits the growth of colon cancer by suppressing cellular proliferation, whilst simultaneously promoting apoptosis.
\end{abstract}

Correspondence to: Dr Yujiang Fang, Department of Microbiology, Immunology and Pathology, Des Moines University College of Osteopathic Medicine, 3200 Grand Avenue, Des Moines, IA 50312, USA

E-mail: yujiang.fang@dmu.edu

*Contributed equally

Key words: interleukin-33, apoptosis, proliferation, colon cancer

\section{Introduction}

Colorectal cancer is the third most commonly diagnosed cancer in the USA, with the second highest cancer-associated mortalities in males and the third highest cancer-associated mortalities in females in USA (1). Although increased screening has decreased morbidity and mortality rates associated with colorectal cancer, 49,190 mortalities were expected in the USA in 2016 (1).

Therapy options for colorectal cancer include neoadjuvant radiotherapy, adjuvant chemotherapy and surgery (2). Immunomodulation has been investigated as a therapeutic agent to increase the efficacy of treatment in patients with cancer. Whilst efficacy of the use of cytokines in colon cancer has been demonstrated, further elucidation and refinement is required (3). Interleukin-33 (IL-33) was discovered in 2005 as a novel member of the IL-1 superfamily (4). IL-33 is expressed by endothelial and epithelial cells. It functions by binding to its primary receptor, ST2, whilst in a complex with the IL-1 receptor accessory protein (3). The role of IL-33 in various host responses to pathogens and allergens has been extensively studied (5); however, the role of IL-33 in cancer remains controversial. A number of studies have indicated that IL-33 has an inhibitory effect on tumor growth (6-8), whilst other studies have determined that it may promote cancer growth (9-11). This indicates that the role of IL-33 in neoplasia is complex and further studies are required to address the exact role of IL-33 in different cancer types. Previously published data demonstrated that IL-33 inhibited proliferation and induced apoptosis of pancreatic cancer cells, and laid the groundwork for the present study (12).

Consequently, the present study was designed to further investigate the direct effect of IL-33 on a frequently studied colon cancer cell line HCT-116 and its possible molecular mechanisms.

\section{Materials and methods}

Tumor cell line. The tumor cell line used for the present study was HCT-116, human colon cancer cells, that were purchased from American Type Culture Collection (Manassas, VA, USA). These HCT-116 cells were grown in Dulbecco's modified 
Eagle's medium (Invitrogen; Thermo Fisher Scientific, Inc., Waltham, MA, USA) at $37^{\circ} \mathrm{C}$ in a $5 \% \mathrm{CO}_{2}$ atmosphere, supplemented with $10 \%$ fetal bovine serum (heat-inactivated) and $1 \%$ penicillin-streptomycin (all from Invitrogen; Thermo Fisher Scientific, Inc.) as previously described (13-17). These cells were cultured in the aforementioned environment until they reached $70 \%$ confluence, upon which they were then subjected to a treatment of IL-33 or medium alone to begin the investigation.

Treatment of HCT-116 cells with IL-33. HCT-116 cells selected to undergo treatment were exposed to $50 \mathrm{ng} / \mathrm{ml}$ IL-33 (Shenandoah Biotechnology, Warwick, PA, USA) over a period of $72 \mathrm{~h}$. Concurrently, the control group was exposed to DMEM (Thermo Fisher Scientific, Inc.) for the same duration of time. The IL-33 concentration and duration were based on the previous work $(12,18,19)$.

Clonogenic survival assay. Following the $72 \mathrm{~h}$ incubation, the HCT-116 cells were detached, manually counted and analyzed using a clonogenic survival assay kit (BioVision, Inc., Milpitas, CA, USA), as previously described $(12,13,18-20)$. The final HCT-116 cell survival results of the study were expressed as a percentage of the total colonies that were counted within the control group.

Immunocytochemistry for proliferating cell nuclear antigen (PCNA). A detailed method for PCNA staining has been previously described $(12,13,18-20)$. To summarize, samples were incubated with $0.1 \%$ saponin (Sigma-Aldrich; Merck KGaA, Darmstadt, Germany) in $1 \%$ bovine serum albumin (Thermo Fisher Scientific, Inc.) and then incubated with polyclonal rabbit antibody for PCNA (sc-7907; dilution 1:100; Santa Cruz Biotechnology, Inc., Dallas, TX, USA) at room temperature for $1 \mathrm{~h}$. The Avidin-Biotin Complex Immunoperoxidase system (Vector Laboratories, Inc., Burlingame, CA, USA) was used to amplify the immunoreactivity of the sample. The samples were then further developed by adding a secondary biotinylated donkey anti-rabbit antibody for $30 \mathrm{~min}$ (711-065-152, 1:500 dilution; Jackson ImmunoResearch Laboratories, Inc., West Grove, PA, USA). NovaRED (Vector Laboratories, Inc.) was then used as the chromogen for staining. Following this, the slides were counterstained with hematoxylin $(2 \mathrm{~g} / \mathrm{l}$; Sigma-Aldrich; Merck KGaA). The PCNA antibody was replaced with an equal amount of normal rabbit IgG (sc-2027; Santa Cruz Biotechnology, Inc.) to establish a negative control, and it was then confirmed that the control slide was negative. Lastly, MetaMorph image analysis software (Molecular Devices LLC, Sunnyvale CA, USA) was used to measure the average immunostaining intensity and the results were expressed as an average integrated immunostaining intensity, which was described previously $(12,13,18-20)$.

Determination of proliferation with the quick cell proliferation assay kit. The Quick Cell Proliferation Assay kit (BioVision, Inc.) was used to quantify HCT-116 cell proliferation in accordance with the manufacturer's protocol, as previously described $(12,13,18-20)$.

Reverse transcription-semi-quantitative polymerase chain reaction ( $R T$-sqPCR). mRNA expression of pro-proliferative molecules [cyclin B, cyclin D, cyclin E, cyclin dependent kinase (CDK)2 and CDK4] and anti-proliferative molecules (p18, p21, p27 and p53) was measured through RT-sqPCR. Similarly, mRNA expression levels of pro-apoptotic molecules [Fas, Fas ligand (FasL), tumor necrosis factor-related apoptosis-inducing ligand (TRAIL), TRAIL receptor (TRAILR) and Bcl-2 associated X (Bax)] and anti-apoptotic molecules [Flice-like inhibitory protein (FLIP), B-cell lymphoma 2 (Bcl-2) and survivin] were also measured with RT-sqPCR. HCT-116 cells were carefully washed with PBS and then homogenized in TRIzol ${ }^{\circledR}$ (Invitrogen; Thermo Fisher Scientific, Inc.). NanoDrop spectrophotometry (Thermo Fisher Scientific, Inc.) was utilized to determine the RNA concentration once the RNA was removed from the cells. A $1 \mu \mathrm{g}$ sample of HCT-116 RNA was used to complete the reverse transcription with a Reverse Transcription system (Cat. no. A3500; Promega Corporation, Madison, WI, USA), as previously described (12,13,18-20). GAPDH was used as an internal control to ensure that equivalent amounts of RNA had been amplified. PCR $(50 \mu \mathrm{l})$ reactions were performed following heating at $94^{\circ} \mathrm{C}$ for $45 \mathrm{sec}, 60^{\circ} \mathrm{C}$ for $60 \mathrm{sec}$ and $72^{\circ} \mathrm{C}$ for $90 \mathrm{sec}$. PCR products were collected prior to the amplifications reaching the plateau phase. Generally, 40 cycles were used. The primer sequences used in the present study are depicted in Table I. The final PCR products were electrophoresed in an agarose gel with the concentration of $2 \%$. Following staining with ethidium bromide $(0.5 \mu \mathrm{g} / \mathrm{ml})$ for $30 \mathrm{~min}$ at room temperature, the PCR products were visualized with UV light and normalized between samples relative to the levels of GAPDH, using an IS-1000 Digital Imaging system (magnification, x10; Thermo Fisher Scientific, Inc.) (17).

Terminal deoxynucleotidyl transferase-mediated dUTP nick-end labeling (TUNEL) staining. TUNEL staining was performed with an ApopTag ${ }^{\circledR}$ kit (EMD Millipore, Billerica, MA, USA) in order to demonstrate the process of apoptosis, as previously described $(12,13,18-20)$. Quantification of the apoptotic cells was also described previously $(12,13,18-20)$. TUNEL+ cancer cells in 3 or 4 randomly selected high-power fields were counted. The resulting TUNEL+ cells were calculated and expressed as a percentage of total cells.

Measurement of caspase-3 activity. The Caspase-3 Colorimetric Assay kit (BioVision, Inc.) was utilized as an additional method to evaluate apoptosis, as described previously $(12,13,18-20)$. The results are expressed as relative caspase-3 activation in cells exposed to IL-33, compared with the caspase- 3 activity of the control cells $(12,13,18-20)$.

Statistical analysis. All data are presented as the mean \pm SEM. An unpaired two-tailed Student's t-test performed using Microsoft Excel 2007 (Microsoft Corporation, Redmond, WA, USA) was used for statistical analysis of the data. $\mathrm{P}<0.05$ was considered to indicate a statistically significant difference. All experiments were repeated for a minimum of two trials.

\section{Results}

Inhibitory effect of IL-33 on the growth of HCT-116 colon cancer cells. Based upon the results of the clonogenic survival 
Table I. Primer sequences used in reverse transcription-polymerase chain reaction.

\begin{tabular}{lll}
\hline Name & \multicolumn{1}{c}{ Sense } & Antisense \\
\hline GAPDH & TGCCGTCTAGAAAAACCTGC & ACCCTGTTGCTGTAGCCAAA \\
p18 & CCTGATCGTCAGGACCCTAA & TTATTGAAGATTTGTGGCTCC \\
p21 & ACACCTAGTTCTACCTCAGGCA & ACTCCCATCATATACCCCT \\
p27 & ACGGGAGCCCTAGCCTGGAGC & TGCCCTTCTCCACCTCTTGCC \\
p53 & TGGCCATCTACAAGCAGTCACA & GCAAATTCCTTCCACTCGGAT \\
Cyclin B & CCATTATTGATCGGTTCATGCAGA & CTAGTGGAGAATTCAGCTGTGGTA \\
Cyclin D & GGATGCTGGAGGTCTGCGAGGAAC & GAGAGGAAGCGTGTGAGGCGGTAG \\
Cyclin E & GGAAGGCAAACGTGACCGTT & GGGACTTAAACGCCACTTAA \\
CDK2 & TTTCTGCCATTCTCATCGG & CTTGGCTTGTAATCAGGCATAGA \\
Fas & ACTTGGGGTGGCTTTGTCTT & GGATGATAGTCTGAATTTTCTCTG \\
FasL & GCCTGTGTCTCCTTGTGA & GCCACCCTTCTTATACTT \\
TRAILR1 & AGAGGGATGGTCAAGGTCAA & GAGTCAAAGGGCACGATGTT \\
TRAIL & AGTCTCTCTGTGTGGCTGTA & TGTCTATCAAGTGCTCATTT \\
Bax & AAGAAGCTGAGCGAGTGT & GGAGGAAGTCCAATGTC \\
FLIP & AATTCAAGGCTCAGAAGCGA & GGCAGAAACTCTGCTGTTCC \\
Bcl-2 & GTGGAGGAGCTCTTCAGGGA & AGGCACCCAGGGTGATGCAA
\end{tabular}

CDK, cyclin dependent kinase; FasL, Fas ligand; TRAIL, tumor necrosis factor-related apoptosis-inducing ligand; TRAILR, TRAIL receptor; Bax, Bcl-2 associated X.
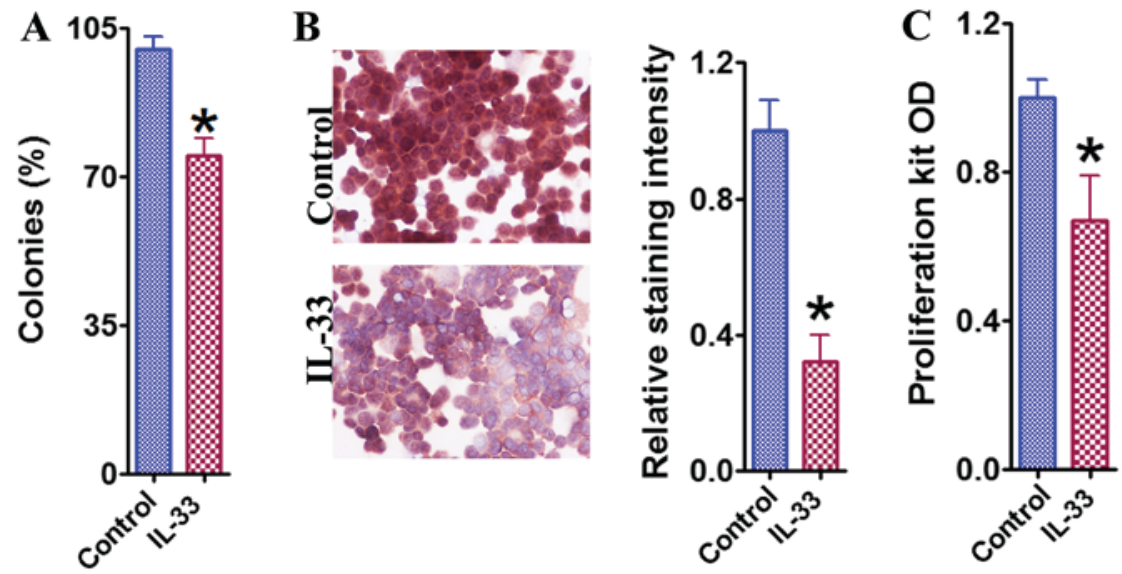

Figure 1. Inhibitory effect of IL-33 on the growth of HCT-116 colon cancer cells. (A) The clonogenic survival assay of HCT-116 cells in the presence and absence of IL-33. The number of colonies was counted and expressed as a percentage of total colonies in the controls. (B) Immunocytochemistry for PCNA of HCT-116 cells in the presence and absence of IL-33. PCNA staining intensity was analyzed and results are expressed as the average integrated staining intensity relative to that in control cells. Original magnification, $x 400$. (C) Cell proliferation evaluated with a proliferation kit in the presence and absence of IL-33. Results are expressed as the mean OD \pm standard error of the mean in each group. ${ }^{*} \mathrm{P}<0.05$, compared with the control. OD, optical density; IL-33, interleukin 33; PCNA, proliferating cell nuclear antigen.

assay, it was noted that the percentage of surviving HCT-116 colonies was significantly lower compared with the control group, following the $72 \mathrm{~h}$ treatment with IL-33 (Fig. 1A). PCNA staining and use of the Quick Cell Proliferation kit further confirmed this observed inhibitory effect of IL-33 (Fig. 1B and C). These results indicate that IL-33 directly inhibited the growth of HCT-116 cells.

IL-33 alters the expression of pro- and anti-proliferative molecules. To investigate the molecular mechanisms, through which IL-33 may impede HCT-116 cell growth and survival, mRNA expression of pro-proliferative molecules [cyclin B, cyclin D, cyclin E, cyclin dependent kinase (CDK)2 and CDK4] and anti-proliferative molecules (p18, p21, p27 and p53) was measured through RT-sqPCR (Fig. 2). The mRNA expression of cyclin $\mathrm{B}$, cyclin $\mathrm{D}$ and $\mathrm{CDK} 2$ was determined to be significantly lower in the IL-33 treated cells compared with the control cells $(\mathrm{P}<0.05)$, whilst the levels of cyclin $\mathrm{E}$ and CDK4 were not significantly altered $(\mathrm{P}>0.05)$. The mRNA expression of the anti-proliferative molecules p21, p27 and p53 was not significantly altered by the treatment $(\mathrm{P}>0.05)$, and there was an unexpected significant decrease in the expression of $\mathrm{p} 18$ in the IL-33 treated cells $(\mathrm{P}<0.05)$. These results indicated that the downregulation of pro-proliferative 

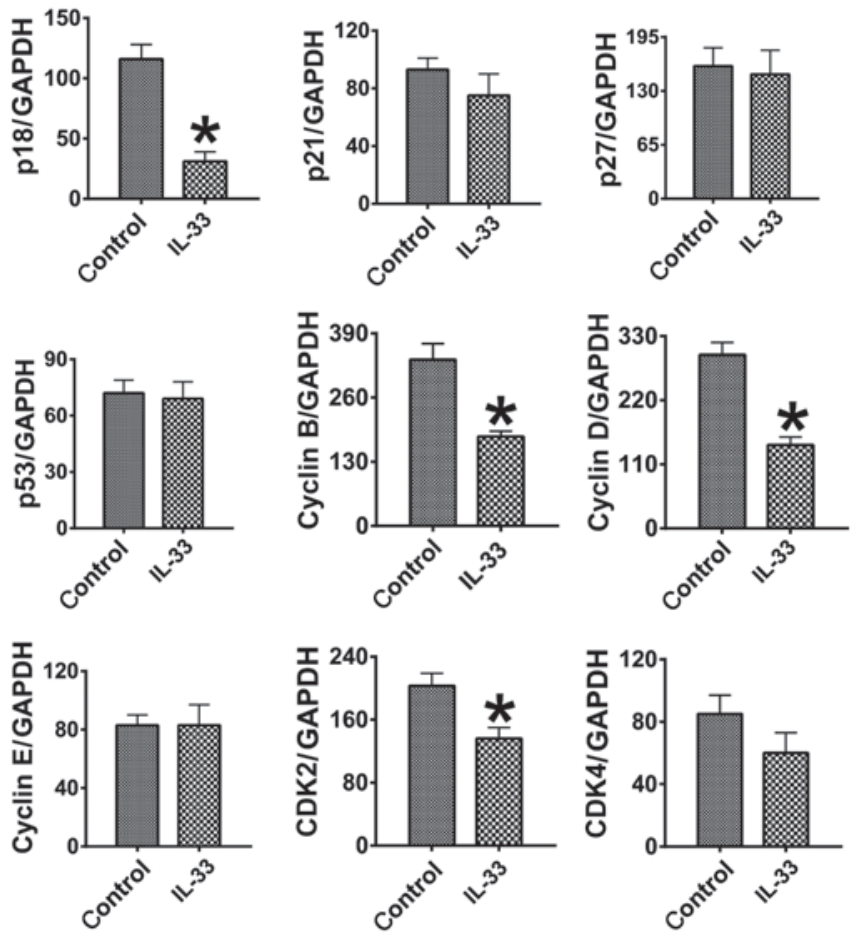

Figure 2. IL-33 alters the expression of pro- and anti-proliferative molecules Results are expressed as the mean ratio of specific molecule densitometric Units/GAPDH \pm standard error of the mean. ${ }^{*} \mathrm{P}<0.05$, compared with the control. CDK, cyclin dependent kinase; IL-33, interleukin-33.

molecules is associated with the inhibitory effect of IL-33 on the proliferation of HCT-116 colon cancer cells.

Promoter effect of IL-33 on apoptosis of HCT-116 colon cancer cells. In addition to proliferative inhibition, IL-33 may also induce a pro-apoptotic effect in HCT-116 cells, as indicated by the TUNEL staining results (Fig. 3A). A significantly higher number of TUNEL+ cells were counted following treatment with IL-33 compared with the control cells $(\mathrm{P}<0.05)$. Caspase-3 activity also demonstrated similar trends (Fig. 3B). These results indicated that IL-33 may exhibit an apoptosis-inducing effect in HCT-116 cells, contributing to the overall inhibitory effect that was observed.

IL-33 alters the expression of pro- and anti-apoptotic molecules. mRNA expression levels of pro-apoptotic molecules [Fas, Fas ligand (FasL), tumor necrosis factor-related apoptosis-inducing ligand (TRAIL), TRAIL receptor (TRAILR) and Bcl-2 associated X (Bax)] and anti-apoptotic molecules [Flice-like inhibitory protein (FLIP), B-cell lymphoma 2 (Bcl-2) and survivin] were measured with RT-sqPCR and used to investigate the molecular mechanisms through which IL-33 may induce apoptosis in HCT-116 cells (Fig. 4). Notably, Fas and TRAIL mRNA expression levels were significantly decreased in the IL-33 treated cells $(\mathrm{P}<0.05)$, although FasL, TRAILR and Bax levels did not significantly change $(\mathrm{P}>0.05)$. Expression levels of FLIP and Bcl-2 were also determined to be significantly decreased in the cells treated with IL-33 $(\mathrm{P}<0.05)$, whilst survivin levels were not altered significantly $(\mathrm{P}>0.05)$. These results indicated that the downregulation of anti-apoptotic molecules, instead of the upregulation of
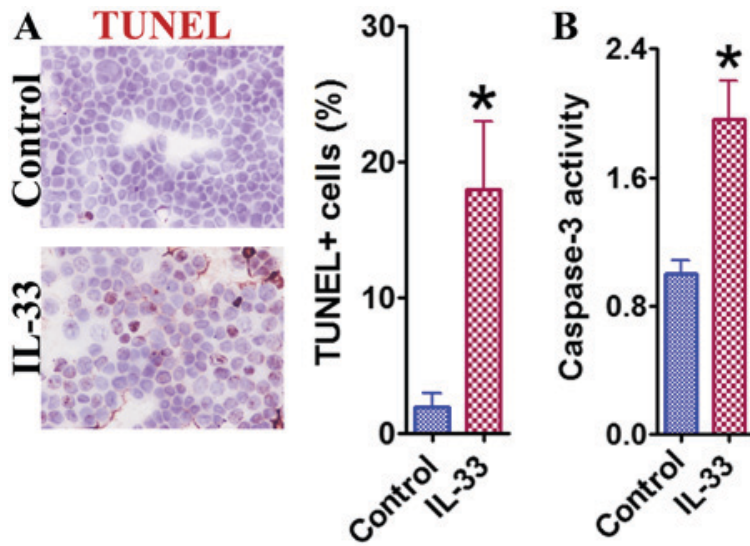

Figure 3. Promoter effect of IL-33 on apoptosis of HCT-116 colon cancer cells. (A) The representative TUNEL staining. TUNEL+ cancer cells in 3-4 randomly selected high-power fields were counted. Original magnification, $\mathrm{x}$ 400. (B) The cellular caspase-3 activity. Results are expressed as mean activity relative to controls \pm standard error of the mean. ${ }^{\star} \mathrm{P}<0.05$, compared with the control. TUNEL, terminal deoxynucleotidyl transferase-mediated dUTP nick-end labeling; IL-33, interleukin 33.
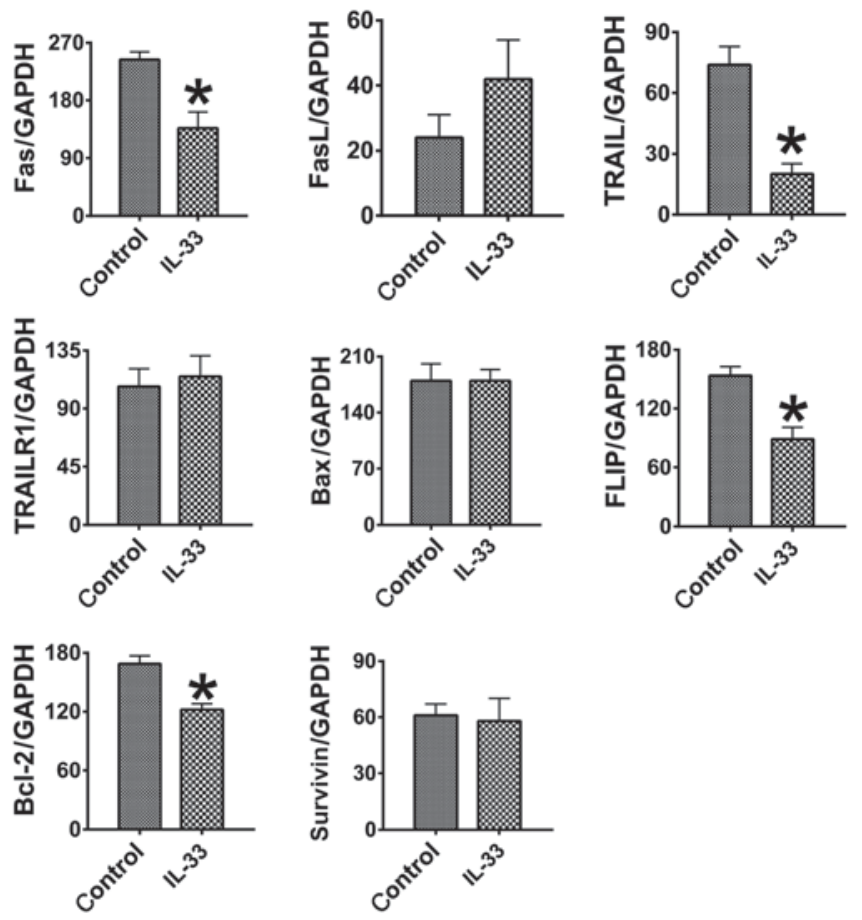

Figure 4. IL-33 alters the expression of pro- and anti-apoptotic molecules. Results are expressed as the mean ratio of specific molecule densitometric Units/GAPDH \pm standard error of the mean. ${ }^{*} \mathrm{P}<0.05$, compared with the control. IL-33, interleukin 33; FasL, Fas ligand; TRAIL, tumor necrosis factor-related apoptosis-inducing ligand; TRAILR, TRAIL receptor; Bax, Bcl-2 associated X.

pro-apoptotic molecules, is associated with the increased apoptosis observed in HCT-116 cells treated with IL-33.

\section{Discussion}

Colorectal cancer remains one of the leading causes of novel cancer diagnoses and cancer-associated mortality in the USA (2). Accordingly, numerous efforts have been undertaken to develop novel treatments to combat this 
prevalent disease. IL-33 has been reported to serve a key role in the initiation of Th2 immunity, inducing the expression of Th2-associated cytokines, and may have been previously overlooked due to its presumed tumorigenic effects that have been well-documented (4). The IL-33 receptor consists of the heterodimeric complex of ST2 and the IL-1 receptor accessory protein, the former of which has been determined to be selectively expressed in Th2 immune responses (3). Additionally, recent studies have determined a higher prevalence in the development of colorectal cancer amongst patients with inflammatory bowel disease, which appeared to be mediated, at least partially, by elevated levels of IL-33 and ST2 expression under the inflammatory state of the disease $(8,9,11,21,22)$. A previous study determined an association between increased IL-33 expression and increased angiogenesis in vivo, indicating that the IL-33 signaling pathway may support carcinogenesis (11). Contrary to these previous studies, the data from the present study indicated that the direct effect of IL-33 on colon cancer was inhibition of proliferation and induction of apoptosis in vitro, indicating that IL-33 may have a complex role in different biological environments. Similar anti-tumorigenic effects of IL-33 have been demonstrated in the previous pancreatic cancer study (12), as well as in a lymphoma study performed with mice models (8).

In the present study, no mRNA or protein expression levels of IL-33 in HCT-116 cells were investigated due to it being demonstrated that IL-33 is undetectable in the HCT-116 colon cancer cell line (23). If IL-33 was determined to be highly expressed in this cancer cell line, then it may be necessary to knock out or block endogenous IL-33 to further confirm the initial data. Although IL-33 is not detectable in HCT-116 colon cancer cells (23), numerous other types of cells, including activated immune cells, fibroblasts and endothelial cells have been reported to produce IL-33 (24). Thus, it is possible that IL-33 produced by these cells may have an effect on the pathogenesis of colon cancer in a biological setting (24).

Tight homeostatic regulation of cellular processes and protein expression is essential for life, and any disruption in these functions may impact cell survival (12-16). Accordingly, the present study investigated the results of a net balance between pro- and anti-proliferative molecules in cells following their exposure to IL-33. mRNA levels of cyclin B, cyclin D, cyclin E, CDK2 and CDK4, which are regarded as pro-proliferative molecules, as well as the levels of p18, p21 and p53, which are regarded as anti-proliferative molecules were specifically examined $(12,18,19,25-27)$. Having determined decreased levels of cyclin B, cyclin D and CDK2, the decreased expression of pro-proliferative molecules was therefore considered as a factor for the observed inhibitory effect of IL-33 on cancer cell proliferation.

The same homeostatic balance may also be described for the proteins regulating apoptosis, and the mRNA levels of pro-apoptotic and anti-apoptotic molecules were examined as well. Fas, FasL, TRAIL, TRAILR1 and Bax promote programmed cell death, and are traditionally categorized as pro-apoptotic molecules, whilst FLIP, Bcl-2 and survivin inhibit cells from undergoing apoptosis, and are traditionally categorized as anti-apoptotic molecules (28). The results indicated that there were decreased levels of FLIP and $\mathrm{Bcl}-2$, which may be responsible for the declined growth and survival of the cancer cells. It is reasonable that these cells, which have decreased levels of self-protective proteins, would have an increased chance of undergoing apoptosis. Therefore, the present study indicated that IL-33 may also disrupt the balance between pro- and anti-apoptotic molecules, resulting in the higher incidence of apoptosis that was observed in the present study.

Notably, it was determined that the anti-proliferative molecule p18, and the pro-apoptotic molecules Fas and TRAIL were significantly downregulated following treatment with IL-33. The overall expected result, given the established roles of these molecules, is that these decreased expression levels should favor cancer cell growth and survival, which directly contradicts these data. Although, the exact pathophysiological significance is unknown, it is possible that this phenomenon may contribute to an adaptive cellular injury response in an attempt to minimize any further cellular damage. As evident by the results of the present study, it may be logical to argue that the overall balance and homeostasis between pro- and anti-proliferative, as well as pro- and anti-apoptotic molecules, instead of individual molecular expression alone, determines the ultimate fate of cancer cells. Similar data have been documented in the previous studies as well, supporting this claim $(12,18,19,25)$.

In conclusion, IL-33 presents a significant anti-tumorigenic effect through inhibition of proliferation and induction of apoptosis in colon carcinoma cells. By disrupting normal protein expression and homeostasis, there may be potential in the utilization of IL-33 as a strategy to treat colon cancer, although further research is required to fully elucidate the mechanisms of IL-33.

\section{Acknowledgements}

The authors thank Mr Brian N. Lifschutz from Des Moines University for his contribution to the language editing.

\section{Funding}

The present study was partially supported by grants for Yujiang Fang (grant nos. IOER 05-14-01, IOER 112-3749 and IOER 112-3104).

\section{Availability of data and materials}

The datasets used and/or analyzed during the current study are available from the corresponding author on reasonable request.

\section{Authors' contributions}

YF conceived the present study. YF, MBN and MRW designed the present study. XC, KL, NJT, DMW, ZZ, CQ, JDM, QB, $\mathrm{HX}$ and YF performed experiments and analyzed the data. YF, MBN, MRW and JDM interpreted the data. XC, NJT, and $\mathrm{ZZ}$ wrote the draft and YF made critical revision. All authors approved the final version of the manuscript.

\section{Ethics approval and consent to participate}

Not applicable. 


\section{Consent for publication}

Not applicable.

\section{Competing interests}

The authors declare that they have no competing interests.

\section{References}

1. Siegel RL, Miller KD and Jemal A: Cancer statistics, 2016. CA Cancer J Clin 66: 7-30, 2016.

2. Brenner H, Kloor M and Pox CP: Colorectal cancer. Lancet 383: 1490-1502, 2014

3. Porta C, Paglino C, Imarisio I and Bonomi L: Cytokine-based immunotherapy for advanced kidney cancer: Past results and future perspectives in the era of molecularly targeted agents ScientificWorldJournal 7: 837-849, 2007.

4. Schmitz J, Owyang A, Oldham E, Song Y, Murphy E, McClanahan TK, Zurawski G, Moshrefi M, Qin J, Li X, et al: IL-33, an interleukin-1-like cytokine that signals via the IL-1 receptor-related protein ST2 and induces T helper type 2-associated cytokines. Immunity 23: 479-490, 2005.

5. Moussion C, Ortega N and Girard JP: The IL-1-like cytokine IL-33 is constitutively expressed in the nucleus of endothelial cells and epithelial cells in vivo: A novel 'alarmin'? PLoS One 3 : e3331, 2008

6. Jovanovic I, Radosavljevic G, Mitrovic M, Juranic VL, McKenzie AN, Arsenijevic N, Jonjic S and Lukic ML: ST2 deletion enhances innate and acquired immunity to murine mammary carcinoma. Eur J Immunol 41: 1902-1912, 2011.

7. Kim W, Kim J, Kim H, Cho H and Kwon B: Association of inhibition of tumor growth with intratumoral hematopoiesis induced by IL-33. J Immunol 186 (Suppl 1): 48.40, 2011.

8. Duault C, Betous D, Bezombes C, Roga S, Cayrol C, Girard JP, Fournié JJ and Poupot M: IL-33-expanded human V $\gamma 9 \mathrm{~V} \delta 2 \mathrm{~T}$ cells have anti-lymphoma effect in a mouse tumor model. Eur J Immunol 47: 2137-2141, 2017.

9. He Z, Chen L, Souto FO, Canasto-Chibuque C, Bongers G, Deshpande M, Harpaz N, Ko HM, Kelley K, Furtado GC and Lira SA: Epithelial-derived IL-33 promotes intestinal tumorigenesis in Apc Min/+ mice. Sci Rep 7: 5520, 2017.

10. Kim JY, Lim SC, Kim G, Yun HJ, Ahn SG and Choi HS: Interleukin-33/ST2 axis promotes epithelial cell transformation and breast tumorigenesis via upregulation of COT activity. Oncogene 34: 4928-4938, 2015.

11. Fang M, Li Y, Huang K, Qi S, Zhang J, Zgodzinski W, Majewski M, Wallner G, Gozdz S, Macek P, et al: IL33 promotes colon cancer cell stemness via JNK activation and macrophage recruitment. Cancer Res 77: 2735-2745, 2017.

12. Fang Y, Zhao L, Xiao H, Cook KM, Bai Q, Herrick EJ, Chen X, Qin C, Zhu Z, Wakefield MR and Nicholl MB: IL-33 acts as a foe to MIA PaCa-2 pancreatic cancer. Med Oncol 34: 23, 2017.

13. Fang Y, DeMarco VG and Nicholl MB: Resveratrol enhances radiation sensitivity in prostate cancer by inhibiting cell proliferation and promoting cell senescence and apoptosis. Cancer Sci 103: 1090-1098, 2012.
14. Fang Y, Yu S and Braley-Mullen H: TGF- $\beta$ promotes proliferation of thyroid epithelial cells in IFN- $\gamma$-/- mice by down-regulation of p21 and p27 via AKT pathway. Am J Pathol 180: 650-660, 2012

15. Fang Y and Braley-Mullen $\mathrm{H}$ : Cultured murine thyroid epithelial cells expressing transgenic Fas-associated death domain-like interleukin-1beta converting enzyme inhibitory protein are protected from Fas-mediated apoptosis. Endocrinology 149: 3321-3329, 2008.

16. Fang Y, Sharp GC, Yagita $\mathrm{H}$ and Braley-Mullen $\mathrm{H}$ : A critical role for TRAIL in resolution of granulomatous experimental autoimmune thyroiditis. J Pathol 216: 505-513, 2008.

17. Fang Y, Wei Y, DeMarco V, Chen K, Sharp GC and Braley-Mullen H: Murine FLIP transgene expressed on thyroid epithelial cells promotes resolution of granulomatous experimental autoimmune thyroiditis in DBA/1 mice. Am J Pathol 170: 875-887, 2007.

18. Fang Y, Chen X, Bai Q, Qin C, Mohamud AO, Zhu Z, Ball TW, Ruth CM, Newcomer DR, Herrick EJ and Nicholl MB: IL-9 inhibits HTB-72 melanoma cell growth through upregulation of p21 and TRAIL. J Surg Oncol 111: 969-974, 2015.

19. Nicholl MB, Chen X, Qin C, Bai Q, Zhu Z, Davis MR and Fang Y: IL-32 $\alpha$ has differential effects on proliferation and apoptosis of human melanoma cell lines. J Surg Oncol 113: 364-369, 2016.

20. Zhu Z, Davidson KT, Brittingham A, Wakefield MR, Bai Q, Xiao $\mathrm{H}$ and Fang Y: Trichomonas vaginalis: A possible foe to prostate cancer. Med Oncol 33: 115, 2016.

21. Zhang Y, Davis C, Shah S, Hughes D, Ryan JC, Altomare D and Peña MM: IL-33 promotes growth and liver metastasis of colorectal cancer in mice by remodeling the tumor microenvironment and inducing angiogenesis. Mol Carcinog 56: 272-287, 2017.

22. Griesenauer B and Paczesny S: The ST2/iL-33 Axis in immune cells during inflammatory diseases. Front Immunol 8: 75, 2017.

23. Lock FE, Babaian A, Zhang Y, Gagnier L, Kuah S, Weberling A, Karimi MM and Mager DL: A novel isoform of IL-33 revealed by screening for transposable element promoted genes in human colorectal cancer. PLoS One 12: e0180659, 2017.

24. Liew FY, Pitman NI and McInnes IB: Disease-associated functions of IL-33: The new kid in the IL-1 family. Nat Rev Immunol 10: 103-110, 2010.

25. Nicholl MB, Ledgewood CL, Chen X, Bai Q, Qin C, Cook KM, Herrick EJ, Diaz-Arias A, Moore BJ and Fang Y: Il-35 promotes pancreas cancer growth through enhancement of proliferation and inhibition of apoptosis: Evidence for a role as an autocrine growth factor. Cytokine 70: 126-133, 2014.

26. Zhang D, Li X, Chen C, Li Y, Zhao L, Jing Y, Liu W, Wang X, Zhang Y, Xia H, et al: Attenuation of p38-mediated miR-1/133 expression facilitates myoblast proliferation during the early stage of muscle regeneration. PLoS One 7: e41478, 2012.

27. Zhu Z, Zhang D, Lee H, Menon AA, Wu J, Hu K and Jin Y: Macrophage-derived apoptotic bodies promote the proliferation of the recipient cells via shuttling microRNA-221/222. J Leukoc Biol 101: 1349-1359, 2017.

28. Griffith TS, Brunner T, Fletcher SM, Green DR and Ferguson TA Fas ligand-induced apoptosis as a mechanism of immune privilege. Science 270: 1189-1192, 1995.

(i) $\ominus$ This work is licensed under a Creative Commons Attribution-NonCommercial-NoDerivatives 4.0 International (CC BY-NC-ND 4.0) License. 